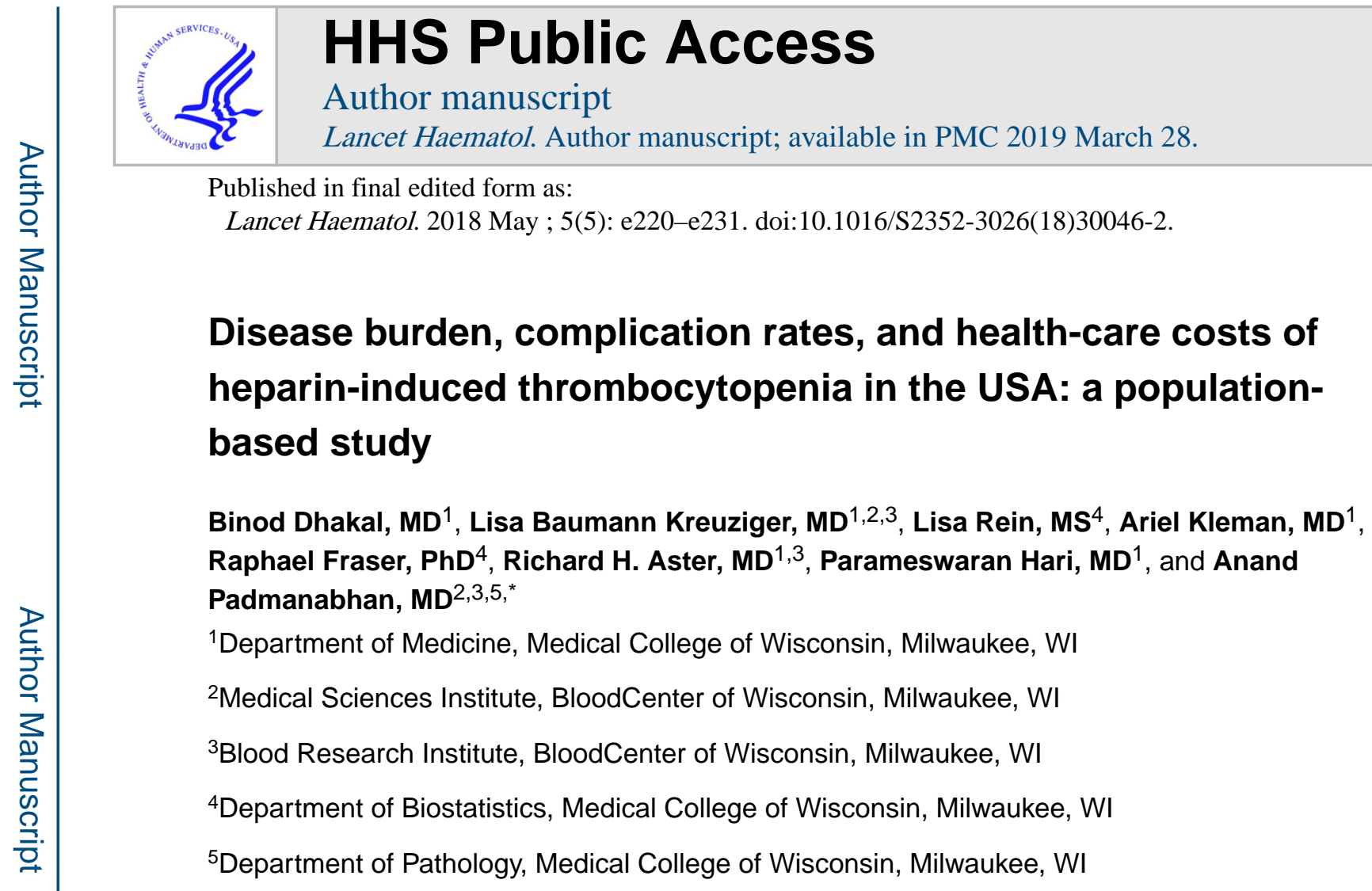

Abstract

*Corresponding author: Anand Padmanabhan MD, PhD, Blood Center of Wisconsin, 8733 Watertown Plank Road, Milwaukee, Wisconsin. anand.padmanabhan@bcw.edu.

Drs. Aster and Hari are Full professors at the Medical College of Wisconsin

Author Contribution:

$\mathrm{BD}, \mathrm{AP}$ and PH conceived the study. LBK and AK performed sensitivity/specificity analysis. LR and RF analyzed the data. RHA provided critical feedback and advice on data interpretation. AP designed the figures. BD wrote the first draft and all authors edited the manuscript and approved its final version.

Research in context

Evidence before this study

We searched PubMed to identify studies describing incidence and complications of HIT and treatments used in the disorder, namely thrombosis, bleeding, amputations and mortality published before Jan 1, 2017 without any date restrictions. Searches were also performed to evaluate incidence of HIT in the following patient populations/diagnoses/procedures: cardiopulmonary bypass, hemodialysis, knee and hip arthroplasty, trauma and gingival/periodontal disease. The incidence and complication rates reported in the literature were noted to be variable, with most of this data obtained from studies with modest sample size. In order to better define disease burden, complication rates and associated costs of HIT, we analyzed a large US inpatient database, the Nationwide Inpatient sample (NIS). Given its large size, the NIS can be particularly helpful in benchmarking incidence and complication rates of infrequent conditions such as HIT.

Added value of this study

This study benchmarks disease burden over a five year period in the US, and details complication rates after analysis of $~ 100,000$ HIT discharges. The incidence of HIT in hospitalized patients was $\sim 1$ in 1500, and the study showed that older patients were at much higher risk for HIT than those less than 50 years of age. Patients undergoing surgery with cardiopulmonary bypass, and hemodialysis had a relatively high incidence of HIT, and, patients with periodontal disease, known to harbor naturally occurring HIT antibodies, had a HIT incidence approximately twice the overall rate. Thrombosis was seen in $\sim 1$ in three HIT discharges, with venous thromboembolism being the most common type. Bleeding was noted in $\sim 6 \%$ of HIT discharges and almost a quarter of patients with HIT and hemorrhage died. Amputations and death were disproportionately high in HIT compared to non-HIT discharges and median length of stay and charges related to hospitalizations were 3-4 higher than in the non-HIT control group.

Conflict of Interest

AP and RHA are listed as inventors on a patent related to HIT diagnostic testing (Patent Number. 9851367 [USPTO]. Pending in other jurisdictions). The other authors have no conflicts of interest to declare.

Corresponding author guarantee

The corresponding author had full access to all the data in the study and had final responsibility for the decision to submit the manuscript for publication. 
Background-Heparin-induced thrombocytopenia (HIT) is a life-and-limb threatening complication of heparin therapy. Its incidence and complication rates have been extrapolated from studies with modest sample size and the outcomes despite current therapeutic interventions are unclear. The goal of this study was to benchmark disease burden, complication rates and costs of HIT in the US.

Methods-We analyzed the Nationwide Inpatient Sample (NIS), a large all-payer inpatient healthcare database in the US, over a recent five-year period (2009-2013). Validation of the HIT ICD-9-CM code was performed by defining the HIT code's (289.84) sensitivity and specificity. The primary outcomes evaluated were the total number of HIT discharges and HIT discharges associated with cardiopulmonary bypass, hemodialysis, hip/knee arthroplasty, trauma and periodontal disease. Other outcomes assessed were the incidence of thrombosis, bleeding, limb/ digit amputation, mortality, length of hospital stay and associated charges.

Findings-Sensitivity and specificity for HIT diagnosis of the HIT ICD-9-CM code were 90.9\% $(57.1-99.5 \%, 95 \%$ CI $)$ and $94.4 \%(91.1-96.6 \%, 95 \%$ CI), respectively. Disease burden was stable at 20,000 discharges/year during the study period. Each year increase in patient age imparted a $2.3 \%$ increased odds of developing HIT. Patients undergoing cardiopulmonary bypass had the highest rates of HIT $0.63 \%$ [0.03\% SE] relative to other patient subgroups examined, while those with hip and knee arthroplasty had low HIT rates, $0.04 \%$ ( $0.004 \%$ SE) and $0.02 \%(0.002 \%$ SE), respectively. The incidence of HIT in gingival/periodontal disease, previously shown to be associated with "naturally occurring" HIT antibodies, was $0.12 \%(0.03 \% \mathrm{SE})$, about twice the overall rate. Thrombosis $(30 \%, 0.4 \% \mathrm{SE})$ and bleeding $(6.2 \%, 0.2 \% \mathrm{SE})$ were common in HIT and a quarter of HIT patients who had hemorrhage died. The adjusted odds of amputation and death were high among HIT compared to non-HIT discharges at aOR 5.1 (4.3-6, 95\% CI) and 4.1 (3.8$4.3,95 \% \mathrm{CI}$ ), respectively. The median length of stay was $>3$-fold and total hospital charges were about $\sim 4$-fold higher in HIT than in non-HIT discharges.

Interpretation-HIT complication rates remain high and are in need of more effective preventative and treatment interventions.

\section{Abstract Presentation:}

Findings contained in this manuscript were presented in part at the American Society of Hematology Annual Meeting and Exposition, Dec 3-6, 2016, San Diego, CA (Use of "Big Data" to define disease burden, complication rates and healthcare costs in patients with heparin induced thrombocytopenia. Oral abstract session 331: Pathophysiology of Thrombosis: Clinical Aspects)

\section{Keywords}

Heparin-induced thrombocytopenia; HIT; heparin; thrombocytopenia; HIT complications; HIT mortality; HIT bleeding; HIT thrombosis; HIT amputations

\section{Introduction}

Heparin induced thrombocytopenia (HIT) is a potentially life-threatening complication that occurs in a subset of heparin-treated patients ${ }^{1}$ and is characterized by platelet-activating antibodies that recognize $\mathrm{PF} 4$-glycosaminoglycan complexes ${ }^{2}$. It is a relatively rare 
condition whose reported incidence and complication rates, based mostly on studies of modest sample size, are variable ${ }^{3,4}$. Reported risks of HIT in patients undergoing hip/knee arthroplasties ${ }^{4-6}$ and hemodialysis ${ }^{7,8}$ have been particularly dissimilar. Reported rates of complications such as thrombosis, bleeding, amputation, and death also differ significantly ${ }^{9-14}$. While some of this variability may reflect heterogeneity of patient populations, surgical interventions, type of heparin used and other factors, an important cause of discrepancies could be that HIT is relatively uncommon, making it difficult to study a large enough number of patients to be representative of the total HIT population.

There are additional areas of uncertainty in HIT. Estimates of the efficacy of non-heparin alternative anticoagulants in HIT are, for the most part, limited to the use of argatroban and comparison of outcomes with historical controls ${ }^{11}$. A recent study prominently questioned the therapeutic efficacy of these treatments in $\mathrm{HIT}^{13}$. There is also a need to characterize HIT risk in specific populations such as those with gingival/periodontal disease, in which "natural" anti-PF4/heparin antibodies are common ${ }^{15}$ and in trauma, where limited data suggest a higher likelihood of HIT $^{16}$. These questions are likely to be addressed more effectively by the use of a large sample set.

To benchmark HIT disease burden, complications and mortality rates more accurately, and to evaluate HIT risk in various patient populations, we analyzed the Nationwide (National) Inpatient Sample (NIS), a database developed for the Healthcare Cost and Utilization Project (HCUP). The NIS is the largest publicly available all-payer inpatient health care database in the United States ${ }^{17}$. Unweighted, it contains data from more than 7 million hospital stays each year and weighted, it estimates more than 35 million hospitalizations nationally ${ }^{17}$. Findings from this study show that the burden of thrombosis, bleeding, amputation, death, and economic costs in HIT is still significant, suggesting a need to develop more effective strategies for diagnosis, prevention and treatment.

\section{Methods:}

\section{Study design and participants}

Adult hospitalizations (patients $\geq 18$ years) in the NIS were analyzed during a recent 5-year period, 2009-2013. The data are de-identified and include a primary diagnosis, the principal problem for which the patient was admitted, as well as secondary diagnosis codes, procedure codes, demographic information, hospital characteristics and related information. Analyses were weighted to provide national estimates. The International Classification of Diseases, Ninth Revision, Clinical Modification (ICD-9-CM) codes of diagnoses and procedures examined in the study are listed in the Appendix (pages 6-7). HIT is almost exclusively a disorder that develops in hospitalized patients following heparin exposure except for rare cases of "spontaneous HIT"18 or infrequent admissions for "delayed-onset HIT"19; thus, discharges with a primary diagnosis of HIT, which reflect these rare presentations or possible miscoded HIT ICD-9-CM, were excluded from this analysis. Also excluded were discharges of patients who had a prior history of thrombosis (see below). After exclusion, survey-weighted domain analysis was conducted to estimate the incidence of HIT among all discharges and in specific patient subsets. Subgroup analysis included patients undergoing procedures or having diagnoses of (1) Cardiopulmonary bypass, (2) Hemodialysis, (3) 
Trauma/injury, (4) Hip arthroplasty, (5) Knee arthroplasty, and (6) Gingival/periodontal disease.

\section{Sensitivity and specificity of the HIT ICD-9-CM code.}

To validate the use of the HIT ICD-9-CM code for identification of patients with HIT, we performed sensitivity/specificity analysis of the HIT ICD-9-CM code (289.84) using patient data from a local hospital (Froedtert Memorial Lutheran Hospital, Milwaukee, WI) covering the latest year of the study period (2013) with approval from the institutional review board of the Medical College of Wisconsin (PRO00028716). Discharges listing IgG-specific PF4/ Polyvinylsulfonate ELISA (PF4 ELISA) testing and/or the HIT ICD-9-CM diagnostic code, 289.84 were evaluated. Encounters with ICD-9-CM history of thrombosis codes (V12.51, V12.52 and V12.55) were excluded; this was done to minimize the likelihood that discharges from patients with a previous history of HIT and associated thrombosis or those with an underlying non-HIT condition that might predispose to thrombosis would be included in the study. $4 \mathrm{Ts}$ scoring ${ }^{20}$ was performed on discharges that met study criteria. "HIT-positive" was defined as patients with an intermediate 4Ts score (4-5) and PF4 ELISA Optical density (OD) $\geq 1$, or high 4Ts score (6-8) and PF4 ELISA OD $\geq 0.4^{21}$.

\section{Outcomes}

The primary outcomes of this study were HIT incidence and complication rates in all hospitalized patients in this cohort. However, heparin exposure during cardiac surgery is a well-recognized risk factor for HIT and was the rationale for including cardiopulmonary bypass as a "positive internal control" for the study. The published data on HIT risk in hemodialysis ${ }^{7,8}$ and orthopedic surgery (hip/knee arthroplasty) ${ }^{4-6}$ have yielded various estimates of incidence and we reasoned it was important to address this issue further using our large dataset. In addition, the recent novel associations between severity of trauma and $\mathrm{HIT}^{16}$, and "naturally-occurring" HIT antibodies and gingival/periodontal diseases ${ }^{15}$ are intriguing and could be further explored in this large study. Clinical outcomes examined included thrombosis, bleeding, amputation, in-hospital mortality, length of stay, and total hospital charges. "Thrombosis" included venous (excluding pulmonary embolism), pulmonary embolism, arterial (excluding "thrombotic stroke"), and thrombotic stroke (cerebral, pre-cerebral artery thrombosis and transient cerebral ischemia). "Bleeding" included intracranial, gastrointestinal, retroperitoneal and unspecified bleeds. Discharges were excluded if these outcomes were listed as primary diagnoses (i.e., the principal problem for which the patient was admitted) to limit the possibility of mere association of one or more of these "outcomes" with HIT that may have developed later on during the admission.

\section{Statistical analysis.}

Survey-weighted domain analysis was conducted to estimate the incidence of HIT among all discharges and in specific patient subsets. Outcomes were compared in discharges with and without HIT using ANOVA for continuous variables and Chi-squared tests for categorical variables and this analysis was also performed within each of the six patient subsets noted above. In subgroup analysis, the non-HIT comparator group was composed of those undergoing procedures or having diagnoses of the diseases of interest but did not develop 
HIT. Length of stay and total hospital charges were log-transformed to satisfy the normality assumption of ANOVA. Multiple logistic regression was used to model each binary clinical outcome with HIT discharge diagnosis as the primary predictor controlling for study year, age, sex, race and hospital teaching status for all outcomes. Additionally, we controlled for platelet transfusion for patient discharges with thrombotic outcomes, given the possible association between platelet transfusion and thrombosis in $\mathrm{HIT}^{22}$. All analyses were performed using SAS software version 9.4 (SAS Institute, Cary, NC) and SAS survey procedures were used for all analyses to properly account for the NIS sampling design. All p-values were 2 -sided and $\mathrm{p}<0.01$ was considered statistically significant.

\section{Results:}

\section{Sensitivity/specificity of HIT ICD-9-CM code for HIT diagnosis.}

Sensitivity and specificity of the HIT ICD-9-CM code, 289.84 were evaluated to validate its use in the study. Patient data from the year 2013 was obtained from a local adult teaching hospital ( $~ 530$ beds) and was screened for encounters with HIT testing (PF4 ELISA) and/or assignment of the HIT ICD-9-CM code as shown in Fig 1A. After exclusion of those with a history of thrombosis, 333 discharges were analyzed. Eighteen false-positive and one falsenegative diagnoses (based on the criteria described in Methods) were made using the HIT ICD-9-CM code. Diagnostic sensitivity and specificity of the HIT ICD-9-CM code were $90.9 \%(57.1-99.5 \%, 95 \% \mathrm{CI})$ and $94.4 \%$ (91.1-96.6\%, 95\% CI), respectively (Fig 1A). The false negative occurred because the encounter was excluded due to the presence of a history of thrombosis ICD-9-CM code (per study design). Due to the limited number of HIT positive cases, this false negative resulted in a wide confidence interval for HIT ICD-9-CM sensitivity.

\section{Disease burden and demographics.}

In the 5-year study period, 109,792 discharges were assigned the HIT ICD-9-CM code as either primary or secondary diagnosis (Fig. 1B). Of these discharges, 10,402 were associated with a history of thrombosis and were therefore excluded. A small number of discharges $(1,824)$ had HIT listed as primary diagnosis and were also excluded. Thus, a total of 97,566 HIT discharges were subjected to analysis. Approximately 150 million $(149,911,247)$ nonHIT discharges during the study period were also included. Basic demographic information is provided in Table 1. Disease burden remained relatively constant over the study period at approximately 20,000/yr. (Fig 2A), with no statistically significant differences noted in the number of discharges in years 2010-2013 relative to the first year of the study (2009). Patients with HIT discharges were significantly older than non-HIT discharges with a median age of 66 vs. 58 yrs. ( $<<0.0001$; Fig 2B) and age imparted significantly higher adjusted odds of developing HIT (adjusted odds ratio [aOR] 1.023 for each year increase in age; $1.022-1.024,95 \% \mathrm{CI}$; $\mathrm{p}<0.0001$ ). In fact, when discharges from patients older than 50 yrs. were compared to $18-50 \mathrm{yr}$. olds, the adjusted odds of developing HIT ranged from 2.7 to 4 (Fig 2C). Females had lower odds of developing HIT overall (aOR 0.775; 0.750-0.802, $95 \%$ CI; Fig 2D), but had a significantly higher odds among the cardiopulmonary bypass (aOR 1.535; 1.382-1.704, 95\% CI) and hemodialysis discharge subset (1.147; 1.069-1.231, 95\% CI) than males. Relative to whites, blacks and members of "other" races had higher 
adjusted odds of HIT (appendix page 2) and discharges from teaching hospitals had a higher rate of HIT than those from non-teaching facilities ( 0.08 vs. $0.05 \%$, p $<0.0001$; appendix page2).

\section{HIT incidence.}

Overall, HIT was identified in $0.065 \%(0.001 \%$ SE) of discharges, approximately 1 in 1500 hospitalizations (Fig 3A). Cardiopulmonary bypass was associated with a relatively high rate of HIT of $0.63 \%(0.03 \% \mathrm{SE})$ followed by hemodialysis $(0.47 \%, 0.01 \% \mathrm{SE})$. Inpatient hemodialysis is frequently administered to critically ill patients who may have a higher risk of HIT than stable patients undergoing outpatient hemodialysis procedures. We used mechanical ventilation as a surrogate for "critical care", and noted that a large number of HIT patients had mechanical ventilation needs $(24.2 \%, 0.7 \%$ SE vs. $9.9 \%, 0.1 \%$ SE in HIT and non-HIT discharges, respectively; data not shown). Trauma/Injury and gingival/ periodontal disease were associated with HIT incidence of $0.09 \%(0.01 \% \mathrm{SE})$ and 0.12 $(0.03 \% \mathrm{SE})$, respectively. Notably, hip and knee arthroplasty were associated with very low HIT rates of $0.04 \%(0.004 \% \mathrm{SE})$ and $0.02 \%(0.002 \% \mathrm{SE})$, respectively (Fig $3 \mathrm{~A})$.

\section{Thrombosis was reported in approximately one-third of all HIT discharges.}

Thrombosis was seen in 30\% ( $0.4 \%$ SE) of HIT discharges compared with only $4.8 \%$ $(0.03 \%$ SE) of non-HIT discharges ( $<<0.0001$; Fig 3B). Venous thrombosis was most common $(17.4 \%, 0.3 \% \mathrm{SE})$, followed by arterial thrombosis $(8.4 \% ; 0.2 \% \mathrm{SE})$, pulmonary embolism $(5.2 \% ; 0.2 \% \mathrm{SE})$ and thrombotic stroke $(4.8 \%, 0.2 \% \mathrm{SE})$. These rates were all significantly higher $(\mathrm{p}<0.0001)$ than in non-HIT discharges (Fig 3B). The adjusted odds of venous thrombosis was high in all HIT discharges (aOR 10.3; 9.8-10.8, 95\% CI), and in each of the six specific patient subpopulations (appendix page 3). Overall, the adjusted odds of pulmonary embolism (appendix page 3), arterial thrombosis (appendix page 3), and thrombotic stroke (appendix page 3) in HIT discharges relative to non-HIT discharges were also high at $9.3(8.6-10,95 \% \mathrm{CI}), 4.7(4.5-5,95 \% \mathrm{CI})$, and $2.2(2-2.3,95 \% \mathrm{CI})$, respectively. Similar to venous thrombosis, the adjusted odds of pulmonary embolism in HIT vs. non-HIT discharges were significantly higher in all evaluable groups (appendix page 3; the gingival/periodontal disease subgroup had too few pulmonary embolism events to be included in the analysis).

\section{Bleeding.}

Bleeding was more common in HIT discharges at $6.2 \%(0.2 \% \mathrm{SE})$ than in non-HIT discharges at $1.9 \%(0.01 \%$ SE; $p<0.0001$; Fig $3 \mathrm{C})$. The most common bleeding site was the gastrointestinal tract at $4.8 \%(0.2 \% \mathrm{SE})$. Intracranial, retroperitoneal, and unspecified bleeding rates were $0.91 \%(0.07 \% \mathrm{SE}), 0.34 \%(0.04 \% \mathrm{SE})$ and $0.28 \%(0.04 \% \mathrm{SE})$, respectively. These rates were significantly higher than those seen in the non-HIT control group ( $\mathrm{p}<0.0001$ for all bleeding outcomes). The adjusted odds of observing intracranial, gastrointestinal, retroperitoneal, or unspecified bleeding in HIT vs. non-HIT discharges were 3.1 (2.6-3.7, 95\% CI; appendix page 4), 2.7 (2.5-2.9, 95\% CI; appendix page 4), 5.5 (4.3-7, $95 \%$ CI; appendix page 4), and 6.2 (4.7-8, 95\% CI; appendix page 4), respectively. 


\section{In-hospital mortality in patients with thrombosis/bleeding.}

Thrombosis rates in non-fatal and fatal HIT were 29\% (0.4\% SE) and 41\% (1.1\% SE), respectively, both significantly higher than in non-HIT discharges ( $<<0.0001$; Fig 4A). Bleeding rates in fatal HIT discharges were approximately triple the rate in non-fatal HIT discharges at $14.7 \%(0.8 \% \mathrm{SE})$ vs. $5.2 \%(0.2 \% \mathrm{SE})$, respectively, and both were significantly higher than in the corresponding non-HIT control group ( $<<0.0001$; Fig 4A). A significant number of patients with HIT and hemorrhage died (24\%, $1.2 \%$ SE) during the hospitalization, while a smaller percent of patients with HIT and thrombosis had the same outcome (14\%, 0.5\% SE; Fig 4B). In-hospital mortality in HIT patients with either thrombosis or hemorrhage was significantly higher than in non-HIT patients $(\mathrm{p}<0.0001$ for both; Fig 4B).

\section{Amputations, in-hospital mortality and economic costs were high with HIT.}

The amputation rate was $0.76 \%(0.06 \% \mathrm{SE})$ vs. $0.12 \%$ (0.001\% SE) in HIT vs. non-HIT discharges $(\mathrm{p}<0.0001)$ and the adjusted odds ratio of amputation overall was high at 5.1 (4.3-6, 95\% CI; appendix page 5). Higher odds of amputation were also seen in the cardiopulmonary bypass and trauma subgroups (appendix page 5). Overall, death occurred in $10.1 \%(0.2 \% \mathrm{SE})$ of HIT discharges compared to $2.1 \%$ (0.01\% SE; $\mathrm{p}<0.0001)$ of discharges without HIT (appendix page 5). Overall, the adjusted odds of death in HIT compared to non-HIT discharges was high (aOR 4.1, 3.8-4.3, 95\% CI; Fig 5A) and this was true for all patient subgroups, except knee arthroplasty (Fig 5A; the gingival/periodontal disease subset was not analyzed due to inadequate number of events). In keeping with the typical development of HIT 5-10 days after heparin exposure, the median time to in-hospital death was significantly longer in HIT than in non-HIT discharges at 12.9 vs. 3.7 days ( $<<0.0001$; Fig 5B). Among those discharged alive, the median length of stay was $>3$-fold longer in those with the HIT ICD-9-CM code than those without (8.9 vs. 2.6 days; $\mathrm{p}<0.0001$; Fig 5C). Prolonged hospitalization and associated costs resulted in $\sim 4$-fold higher median hospitalization charges in HIT compared to non-HIT discharges $(\$ 83,072$ vs. $\$ 21,360 ; \mathrm{p}<0.0001$; Fig 5D).

\section{Discussion}

HIT is classified as a rare disease by the National Institutes of Health ${ }^{23}$. Large databases such as the NIS can be particularly helpful in benchmarking incidence and complication rates of infrequent conditions. Assessments of HIT utilizing "big data" have been performed in three studies reported previously ${ }^{24-26}$. Two of these were limited in scope, being focused solely on cardiac procedures ${ }^{24,25}$. The third study ${ }^{26}$ assessed the disease burden of HIT in a 3 -yr period and examined thrombosis and mortality outcomes in affected patients, but did not exclude patients with a prior history of thrombosis or control for platelet transfusions, a risk factor for thrombosis ${ }^{22}$. To our knowledge, the current study is the first large-scale analysis of bleeding and amputation risks in HIT patients and in specific patient subgroups such as those undergoing hemodialysis, experiencing trauma or having periodontal disease. We also examine, for the first time, to our knowledge, sensitivity and specificity of the HIT ICD-9-CM code as a way of validating the findings. 
The incidence of HIT in hospitalized US patients was $\sim 1$ in 1500, higher than estimates of $\sim 1$ in 5000 reported in some expert reviews ${ }^{27}$. The relatively stable disease burden observed during the study period was surprising because there has been increased use in recent decades of low molecular weight heparin (LMWH), thought to be less likely than unfractionated heparin (UFH) to cause HIT. However, the aOR of HIT in the latest year of the study period relative to the earliest was 0.867 and trended towards significance $(0.772-$ $0.974,95 \% \mathrm{CI}, \mathrm{p}=0.016$ ). This study demonstrates that older age is significantly associated with HIT. It is possible that this positive correlation reflects greater heparin exposure in older than in younger patients rather than a biological effect of age per se on HIT development, but this remains to be tested. Among specific patient groups examined, patients undergoing surgery with cardiopulmonary bypass ("on-pump" procedures that almost always use heparin) and hemodialysis had a relatively high incidence of HIT. While heparin exposure during cardiac surgery is a well-recognized risk for HIT development, the data on HIT risk in hemodialysis is highly variable but considered to be low by some experts. Our findings indicate that the incidence of HIT in patients on inpatient hemodialysis is relatively high $(0.47 \%, 0.01 \% \mathrm{SE})$ and that a significant proportion of these patients were critically ill. Hence these rates may not be applicable to HIT risk in the outpatient setting where hemodialysis is performed on relatively stable patients. To our knowledge, we have shown for the first time that patients with periodontal disease, known to be at higher risk for naturally occurring HIT antibodies ${ }^{15}$, have a HIT incidence approximately twice the overall rate. If confirmed by further studies, these findings suggest that closer monitoring of heparin-treated patients with periodontal disease may be indicated. We found rates of HIT in hip and knee arthroplasty to be low and could reflect greater use of LMWH during the study period.

Thrombosis, a frequent and potentially fatal complication of HIT, was seen in $\sim 1$ in three HIT discharges, with venous thromboembolism being most common type. HIT, in itself, typically does not cause bleeding ${ }^{27}$, and when this complication occurs, it is usually caused by anticoagulants used to treat or prevent thrombosis. Among the limitations, this study cannot distinguish between bleeding directly attributed to HIT (likely very rare) and bleeding that is likely due to the use of non-heparin alternative anticoagulants. We observed a $\sim 6 \%$ bleeding rate among HIT discharges, consistent with previous estimates of bleeding at $\sim 7 \%^{11}$ for argatroban and $\sim 8 \%{ }^{28}$ for bivalirudin. Of note, we found that almost a quarter of patients with HIT and hemorrhage died. While this does not prove causality, it is a concerning finding that merits additional investigation. Although the adjusted odds of amputations was high in HIT relative to non-HIT discharges, the rate of $0.76 \%(0.06 \% \mathrm{SE})$ may underestimate the true amputation rate due at least to a couple of reasons: 1) Patient death (before amputation could be performed), and 2) Patient discharge with limb ischemia to a rehabilitation facility. In the latter instance, amputation, if necessary, would have been performed in-hospital in a subsequent hospitalization and thus captured by the NIS in a distinct non-HIT encounter. Finally, one in every ten HIT patients died in hospital, and length and costs related to hospitalizations were high, reflecting significant morbidity and economic burden associated with this condition.

Other limitations of our study require comment. Coding errors are known to exist in administrative databases. We excluded patients with a primary diagnosis of any of the 
outcomes studied (e.g. thrombosis) to minimize the likelihood of these outcomes being coincidentally associated with HIT that developed during the admission. However, it is well known that HIT can develop after therapeutic use of heparin to treat thrombosis; hence the study design could potentially underestimate HIT incidence. In addition, although we assessed the sensitivity and specificity of the HIT diagnostic code using data acquired independently from a local hospital, it was unfeasible to evaluate all codes (e.g. outcomes) similarly. It appears that sensitivity/specificity of ICD-9-CM bleeding codes are high $(>90 \%)^{29}$. We were unable to categorize bleeding as major vs. minor defined by widely accepted criteria (such as from ISTH) due to difficulties in extracting relevant information to make these determinations. Finally, the NIS does not include medication information and cannot be used to define the incidence of HIT among patients exposed to UFH vs. LMWH, and outcomes associated with more recent therapies (used after 2014) would not have been captured.

Despite these shortcomings, the comprehensive and large size of the dataset used allows for clinically important outcomes to be evaluated in this relatively uncommon condition and avoids the biases associated with single and multicenter studies of modest sample size. It provides a "real world" picture of the HIT burden spread across a wide spectrum of inpatient facilities, with associated complication rates, mortality, and resource utilization information. We believe this information will help benchmark HIT burden and complication rates and can assist with development of public health interventions such as the recently applied singlecenter "avoid heparin" protocol to decrease HIT incidence ${ }^{30}$. The complication rates noted in our study are a somber reminder of the severe morbidity and mortality of HIT despite decades of basic and clinical research on this disease and emphasize the need for novel diagnostics and treatments, particularly in severe forms of the disease.

\section{Supplementary Material}

Refer to Web version on PubMed Central for supplementary material.

\section{Acknowledgments:}

We would like to thank Aaron Tobian, MD (Johns Hopkins University) for helpful discussions, Alex Buschmann (Medical College of Wisconsin) for assistance with ICD-9-CM coding information and Tom Chelius, MS for help with the NIS dataset. AP was supported by a Mentored Clinical Scientist Research Career Development Award (NIH grant HL133479). We would also like to acknowledge the Healthcare Cost and Utilization Project (HCUP) and its data partners (https://www.hcup-us.ahrq.gov/db/hcupdatapartners.jsp) for enabling us to conduct this study.

Funding

AP was supported by a Mentored Clinical Scientist Research Career Development Award (NIH grant HL133479).

Role of Funding Source

The funding source did not have any role in study design, data collection, analysis, interpretation, summarizing the data or decision to submit the manuscript for publication.

\section{References:}

1. Cuker A, Cines DB. How I treat heparin-induced thrombocytopenia. Blood 2012;119(10):22092218. [PubMed: 22246036] 
2. Visentin GP, Ford SE, Scott JP, Aster RH. Antibodies from patients with heparin-induced thrombocytopenia/thrombosis are specific for platelet factor 4 complexed with heparin or bound to endothelial cells. J Clin Invest 1994;93(1):81-88. [PubMed: 8282825]

3. Morris TA, Castrejon S, Devendra G, Gamst AC. No difference in risk for thrombocytopenia during treatment of pulmonary embolism and deep venous thrombosis with either low-molecular-weight heparin or unfractionated heparin: a metaanalysis. Chest 2007;132(4):1131-1139. [PubMed: 17646239]

4. Warkentin TE, Levine MN, Hirsh J, et al. Heparin-induced thrombocytopenia in patients treated with low-molecular-weight heparin or unfractionated heparin. N Engl J Med 1995;332(20):13301335. [PubMed: 7715641]

5. Craik JD, Cobb AG. Heparin-induced thrombocytopenia following hip and knee arthroplasty. Br J Haematol 2013;161(2):255-261. [PubMed: 23406088]

6. Smythe MA, Koerber JM, Mattson JC. The incidence of recognized heparin-induced thrombocytopenia in a large, tertiary care teaching hospital. Chest 2007;131(6):1644-1649. [PubMed: 17400685]

7. Ferreira JA, Johnson DW. The incidence of thrombocytopenia associated with continuous renal replacement therapy in critically ill patients. Ren Fail 2015;37(7):1232-1236. [PubMed: 26108135]

8. Yamamoto S, Koide M, Matsuo M, et al. Heparin-induced thrombocytopenia in hemodialysis patients. Am J Kidney Dis 1996;28(1):82-85. [PubMed: 8712226]

9. Warkentin TE, Kelton JG. A 14-year study of heparin-induced thrombocytopenia. Am J Med 1996;101(5):502-507. [PubMed: 8948273]

10. Almeida JI, Coats R, Liem TK, Silver D. Reduced morbidity and mortality rates of the heparininduced thrombocytopenia syndrome. J Vasc Surg 1998;27(2):309-314; discussion 315-306. [PubMed: 9510285]

11. Lewis BE, Wallis DE, Berkowitz SD, et al. Argatroban anticoagulant therapy in patients with heparin-induced thrombocytopenia. Circulation 2001;103(14):1838-1843. [PubMed: 11294800]

12. Haas $\mathrm{S}$, Lewis B. Identifying patient- and treatment-related factors related to amputation risk in cases of heparin-induced thrombocytopenia treated with argatroban. Int Angiol 2011;30(6):541546. [PubMed: 22233615]

13. Kuter DJ, Konkle BA, Hamza TH, et al. Clinical outcomes in a cohort of patients with heparininduced thrombocytopenia. Am J Hematol 2017;92(8):730-738. [PubMed: 28388835]

14. Baroletti S, Hurwitz S, Conti NA, Fanikos J, Piazza G, Goldhaber SZ. Thrombosis in suspected heparin-induced thrombocytopenia occurs more often with high antibody levels. Am J Med 2012;125(1):44-49. [PubMed: 22075045]

15. Greinacher A, Holtfreter B, Krauel K, et al. Association of natural anti-platelet factor 4/heparin antibodies with periodontal disease. Blood 2011;118(5):1395-1401. [PubMed: 21659541]

16. Lubenow N, Hinz P, Thomaschewski S, et al. The severity of trauma determines the immune response to $\mathrm{PF} 4 /$ heparin and the frequency of heparin-induced thrombocytopenia. Blood 2010;115(9):1797-1803. [PubMed: 19965682]

17. https://www.hcup-us.ahrq.gov/nisoverview.jsp Accessed 8/4/17.

18. Warkentin TE, Basciano PA, Knopman J, Bernstein RA. Spontaneous heparin-induced thrombocytopenia syndrome: 2 new cases and a proposal for defining this disorder. Blood 2014;123(23):3651-3654. [PubMed: 24677540]

19. Rice L, Attisha WK, Drexler A, Francis JL. Delayed-onset heparin-induced thrombocytopenia. Ann Intern Med 2002;136(3):210-215. [PubMed: 11827497]

20. Lo GK, Juhl D, Warkentin TE, Sigouin CS, Eichler P, Greinacher A. Evaluation of pretest clinical score (4 T's) for the diagnosis of heparin-induced thrombocytopenia in two clinical settings. J Thromb Haemost 2006;4(4):759-765. [PubMed: 16634744]

21. Cuker A, Crowther M. 2013 Clinical Practice Guideline on the Evaluation and Management of Adults with Suspected Heparin-Induced Thrombocytopenia (HIT) wwwhematologyorg/Clinicians/ Guidelines-Quality/Quick-Ref/529aspx 2013.

22. Goel R, Ness PM, Takemoto CM, Krishnamurti L, King KE, Tobian AA. Platelet transfusions in platelet consumptive disorders are associated with arterial thrombosis and in-hospital mortality. Blood 2015;125(9):1470-1476. [PubMed: 25588677] 
23. https://rarediseases.info.nih.gov/ Accessed 8/4/17.

24. Seigerman M, Cavallaro P, Itagaki S, Chung I, Chikwe J. Incidence and outcomes of heparininduced thrombocytopenia in patients undergoing cardiac surgery in North America: an analysis of the nationwide inpatient sample. J Cardiothorac Vasc Anesth 2014;28(1):98-102. [PubMed: 24295719]

25. Telila T, Akintoye E, Ando T, et al. Incidence and Outcomes of Heparin-Induced Thrombocytopenia in Patients Undergoing Transcatheter Aortic Valve Replacement. Am J Cardiol 2017;120(2):300-303. [PubMed: 28576265]

26. Pathak R, Bhatt VR, Karmacharya P, Aryal MR, Donato AA. Medical and Economic Burden of Heparin-Induced Thrombocytopenia: A Retrospective Nationwide Inpatient Sample (NIS) Study. J Hosp Med 2017;12(2):94-97. [PubMed: 28182804]

27. Greinacher A CLINICAL PRACTICE. Heparin-Induced Thrombocytopenia. N Engl J Med 2015;373(3):252-261. [PubMed: 26176382]

28. Joseph L, Casanegra AI, Dhariwal M, et al. Bivalirudin for the treatment of patients with confirmed or suspected heparin-induced thrombocytopenia. J Thromb Haemost 2014;12(7):1044-1053. [PubMed: 24766902]

29. Delate T, Jones AE, Clark NP, Witt DM. Assessment of the coding accuracy of warfarin-related bleeding events. Thromb Res 2017;159:86-90. [PubMed: 29035718]

30. McGowan KE, Makari J, Diamantouros A, et al. Reducing the hospital burden of heparin-induced thrombocytopenia: impact of an avoid-heparin program. Blood 2016;127(16):1954-1959.

[PubMed: 26817956] 


\section{Implications of all the available evidence}

The large size of the dataset used for analysis allowed for clinically important outcomes to be evaluated in this relatively infrequent disorder. HIT complication rates remain high and are in need of more effective interventions. Incidence and outcomes benchmarked here are expected to help with evaluation of the impact of newer diagnostic, preventative and treatment approaches in HIT. 


\section{Systematic Review}

a) Did you do a systematic review as part of the planning for this trial?

Author reply: We did not perform a systematic review as a part of the planning for this trial.

b) If yes, please provide details of databases searched, search terms used, any restrictions on search, etc. How did you select and combine the evidence? c) If not, how did you know it was appropriate to do the trial?

Author reply: Based on our extensive knowledge of the published HIT literature, we are acutely aware that disease burden and complications rates in HIT are mainly based on data from studies of small/modest size. We decided to perform this study using a large number of HIT cases from a national database to more confidently benchmark disease burden and complication rates.

d) What is the existing evidence in this area of research, and how did you identify it?

Author reply: The existing evidence suggests that the incidence of HIT may be as low as 1 in 5000 hospitalizations, yet as high as $\sim 3 \%$ in some patient groups. Similar variable estimates exist in complication rates of HIT such as thrombosis. These reports were identified from MEDLINE. 


\section{Interpretation}

a) How does the present trial fit into that evidence?

Author reply: The present study analyzed $\sim 100,000$ HIT and $~ 150$ million non-HIT discharges using a national medical administrative database. This approach was complementary to other approaches in HIT including case reports, cohort studies and the few randomized controlled trials that have provided most of the information that makes up current evidence.

b) What do the findings of all the trials and the present trial mean?

Author reply: The combined findings suggest that the morbidity and mortality burden of HIT remains significant, despite advances in diagnostic and treatment strategies in this dangerous disorder.

c) What are the clinical implications of your findings? What should clinicians do now?

Author reply: Clinicians should be acutely aware of HIT and its treatment complications, including bleeding, and should keep abreast of the constantly changing literature. We also hope that the information provided will inspire development of novel therapies, especially for severely afflicted patients. 


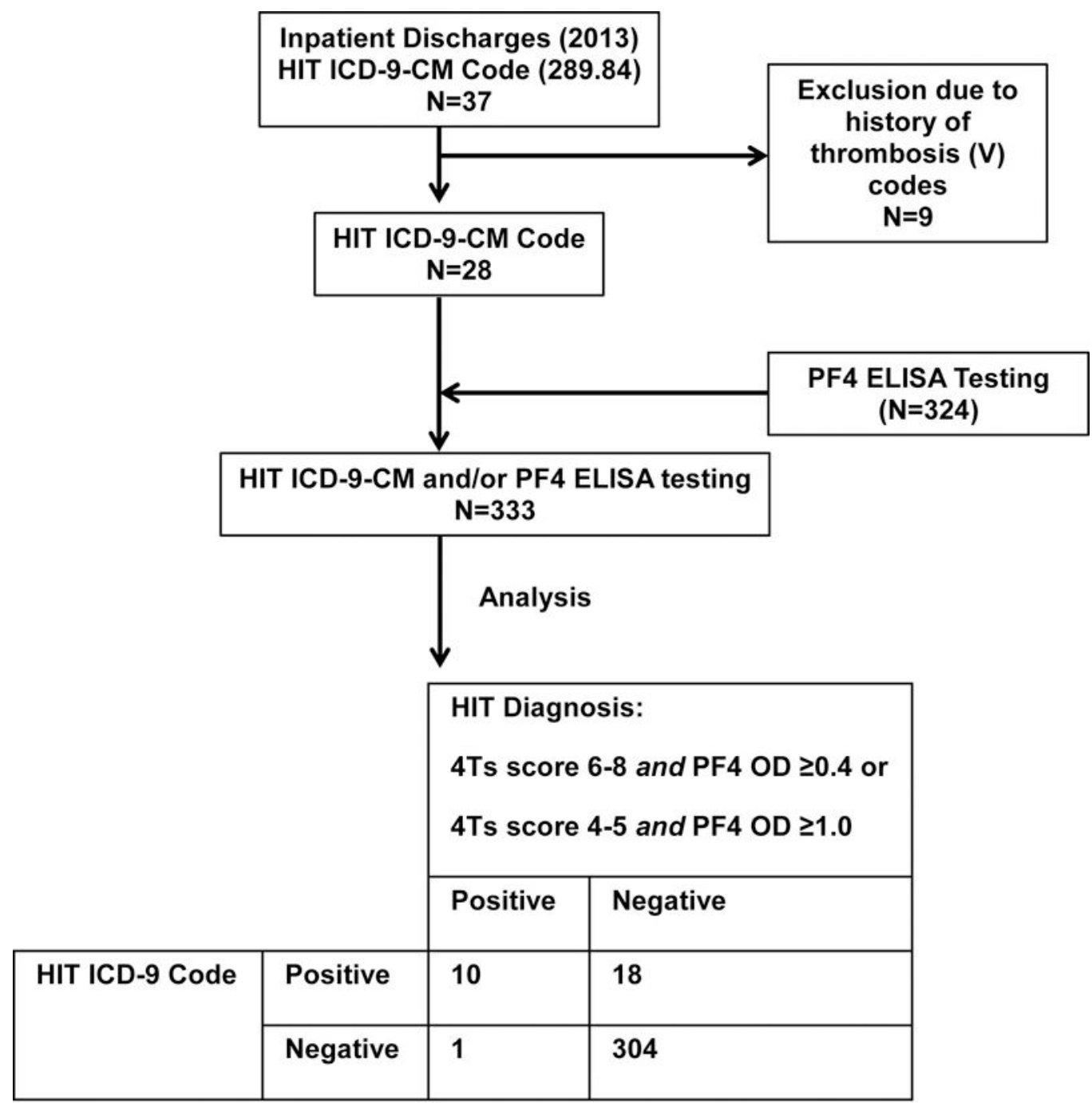

Sensitivity: $90.9 \%$ (57.1-99.5\%, $95 \% \mathrm{Cl})$

Specificity: $94.4 \%(91.1-96.6 \%, 95 \% \mathrm{Cl})$ 


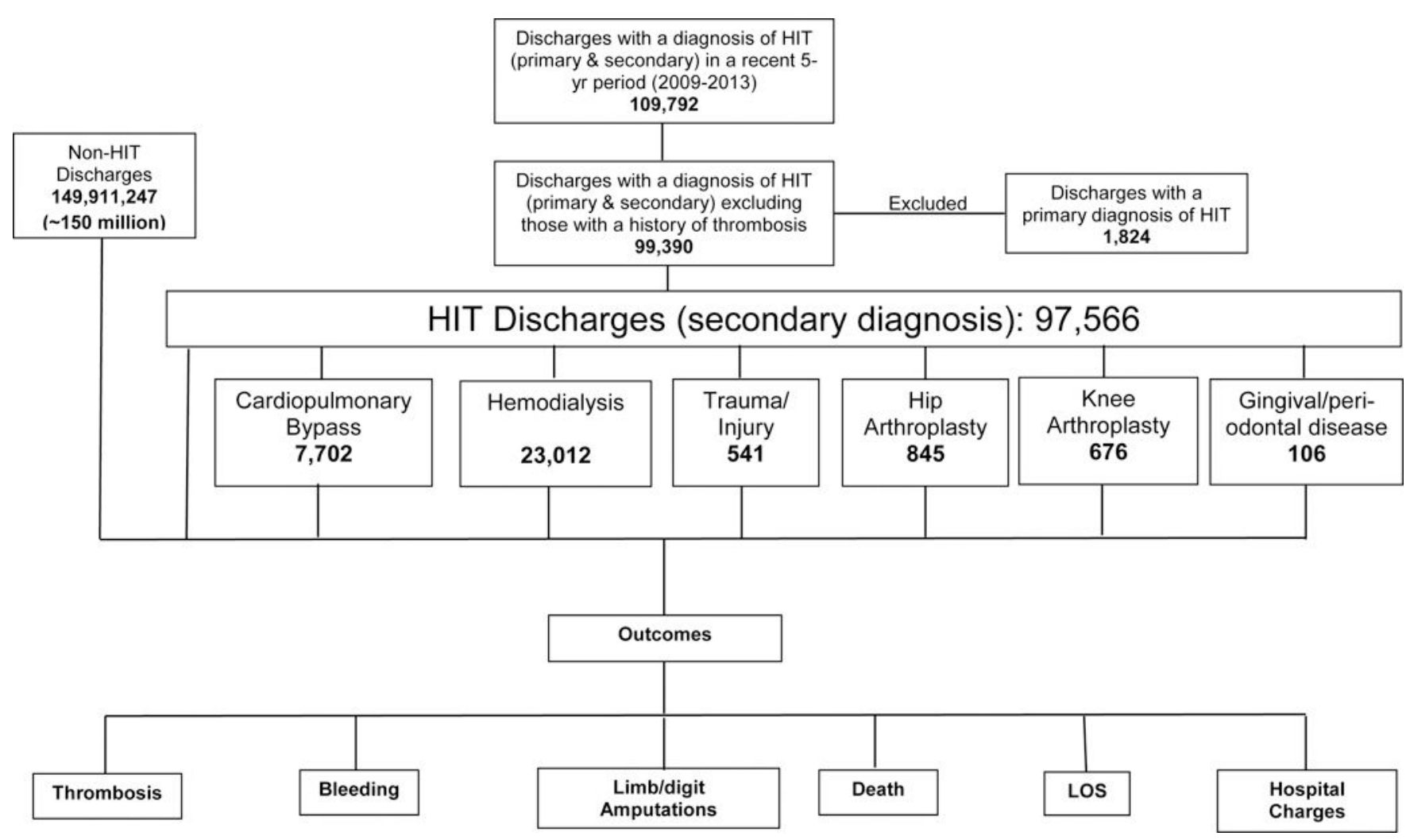

Figure 1. HIT ICD-9-CM code and Study Design.

(A) Sensitivity/specificity analysis of HIT ICD-9-CM code. Discharges from a local hospital during the most recent year of the study period were analyzed as described. (B) Study Design. Thrombosis, bleeding, amputations, death, hospital length of stay (LOS) and hospital charges were evaluated in non-HIT discharges and in discharges with a secondary diagnosis of HIT after exclusion of those with history of thrombosis. These outcomes were also evaluated in discharge subgroups with cardiopulmonary bypass, hemodialysis, hip/knee arthroplasty procedure codes, and diagnosis of trauma/injury and gingival/periodontal disease. 
2A

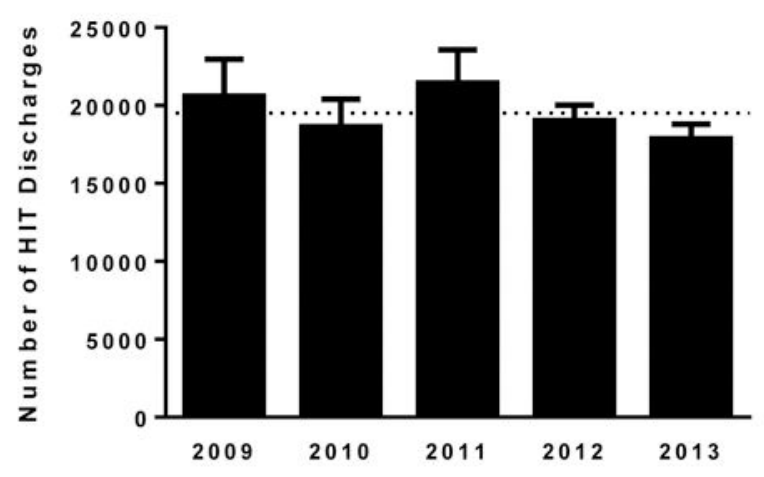

$2 \mathrm{C}$

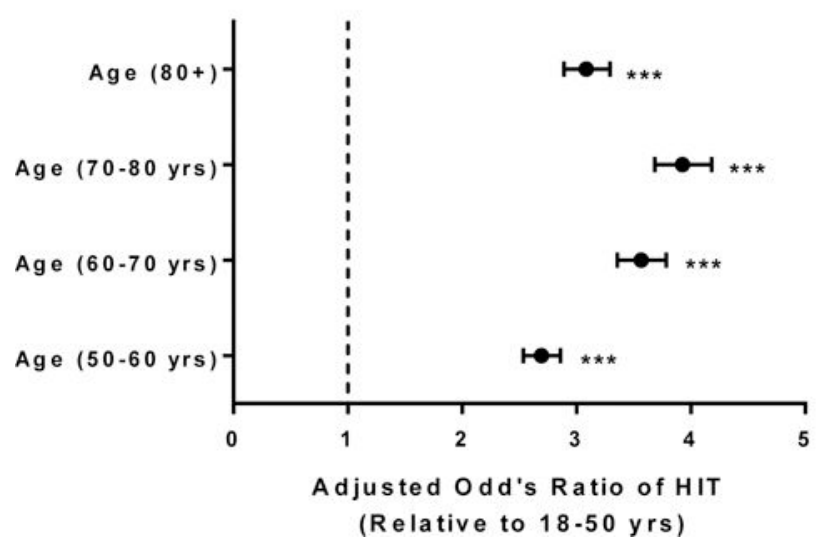

$2 \mathrm{~B}$

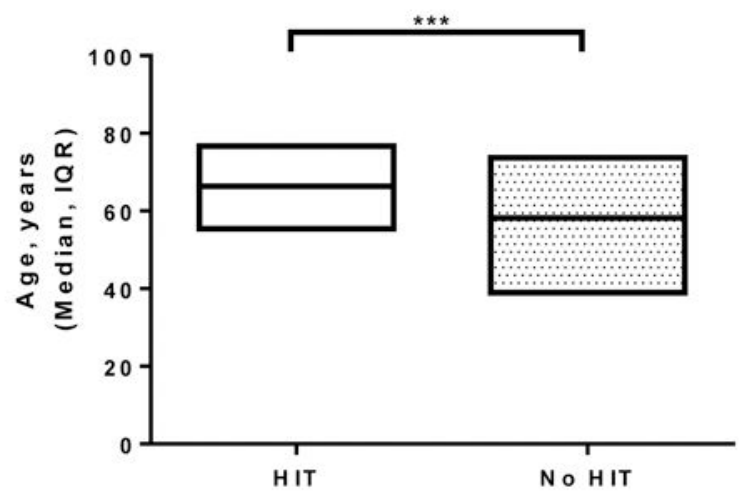

Figure 2. HIT burden and associations with Age and Sex.

(A) The HIT burden was stable at $\sim 20,000$ per year during the study period. The abscissa denotes year of study and ordinate shows the weighted frequency of HIT. Error bars denote $95 \%$ CIs. The horizontal dotted line indicates the mean of weighted frequencies over the 5-year study period. (B) High Median age in HIT discharges. Abscissa denotes HIT vs. non-HIT discharges and the ordinate depicts median age of patient and interquartile range (IQR). (C-D) Adjusted odds ratio of HIT was significantly higher in older patients (C) and males overall (D). $* * *$ denotes $\mathrm{p}<0.0001$. ns-not significant. CPB- Cardiopulmonary bypass. 
A

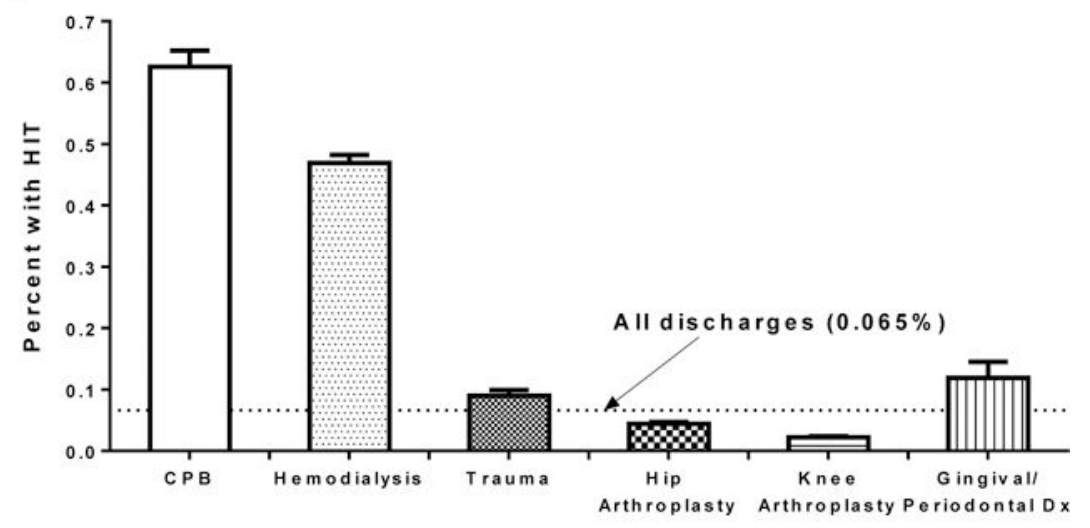

B

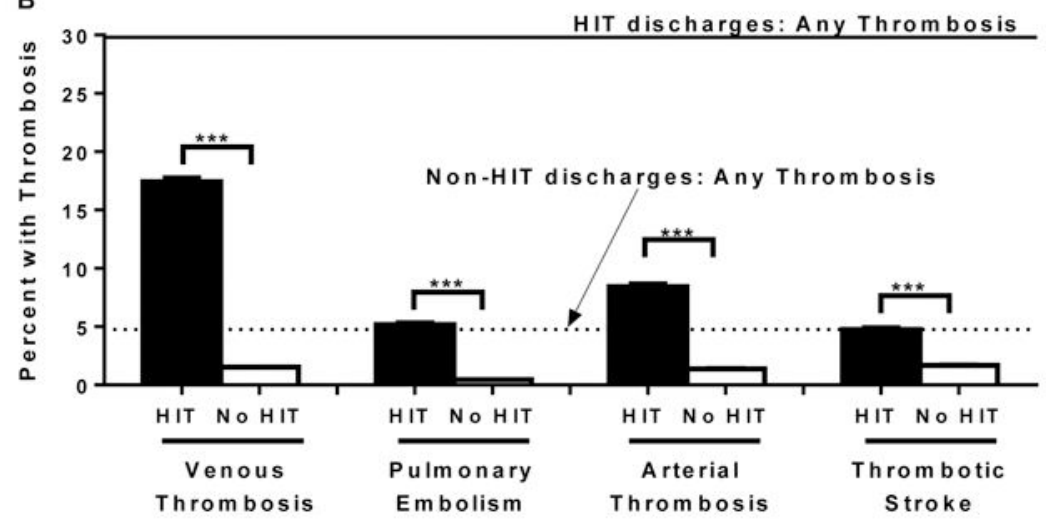

C

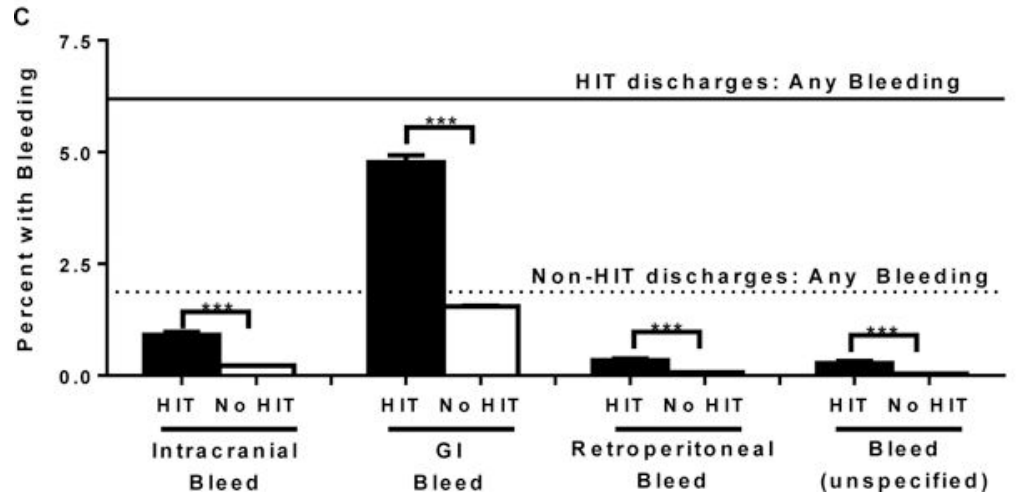

Figure 3. HIT in discharge subsets, and Thrombosis/Bleeding complication rates (A) Incidence of HIT in discharge subsets. Abscissa denotes patient subgroup by diagnosis/procedure type. Percent of discharges within each subset that had a diagnosis of HIT are shown on the ordinate. Error bars denote standard error (SE). The horizontal dotted line indicates percent of all discharges that had a diagnosis of HIT. (B) Thrombosis in patients assigned a discharge diagnosis of HIT. Abscissa denotes type of thrombosis and ordinate shows the percent of discharges with thrombosis codes. Error bars denote standard error (SE). The solid and dotted horizontal lines indicate thrombosis rates in HIT and non- 
HIT discharges, respectively. (C) Bleeding in patients assigned a discharge diagnosis of HIT. Abscissa denotes type of bleeding and ordinate shows the percent of discharges with bleeding codes. Error bars denote standard error (SE). The solid and dotted horizontal lines indicate bleeding rates in HIT and non-HIT discharges, respectively. 


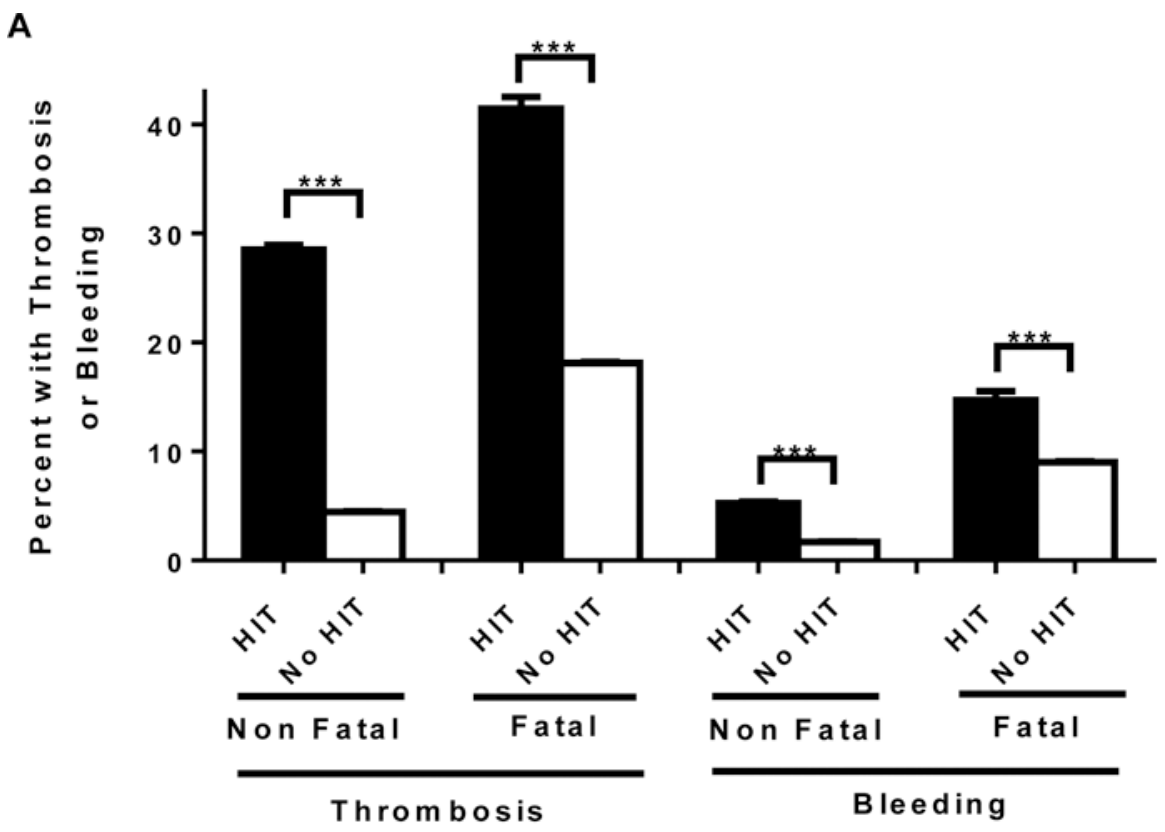

B

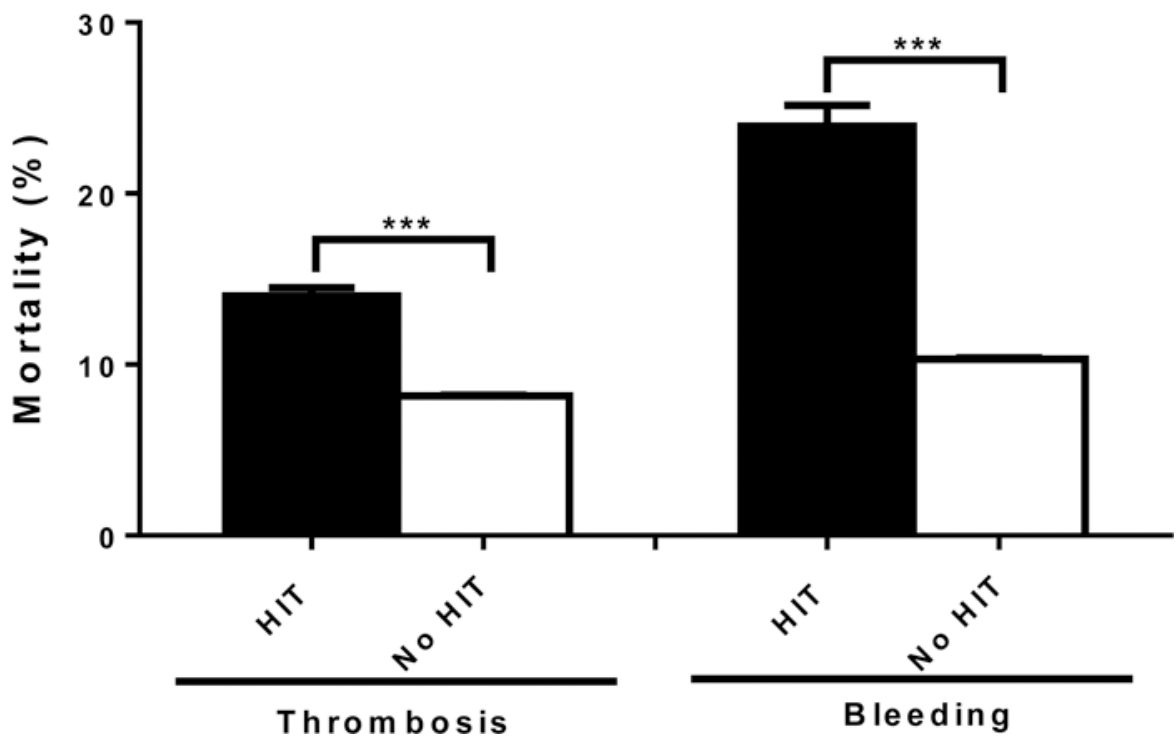

Figure 4. In-hospital mortality and association with Thrombosis/Bleeding.

(A) Thrombosis and bleeding were more common in patients who had a fatal outcome. Abscissa denotes fatal and non-fatal outcomes in HIT and non-HIT discharges. The ordinate shows the percent of discharges with thrombosis or bleeding codes. Error bars denote standard error (SE). (B) In-hospital mortality in patients with bleeding or thrombosis. Abscissa denotes thrombosis or bleeding in HIT and non-HIT discharges. The ordinate shows the percent of discharges that reported patient death. Error bars denote standard error (SE). $* * *$ denotes $\mathrm{p}<0.0001$. 


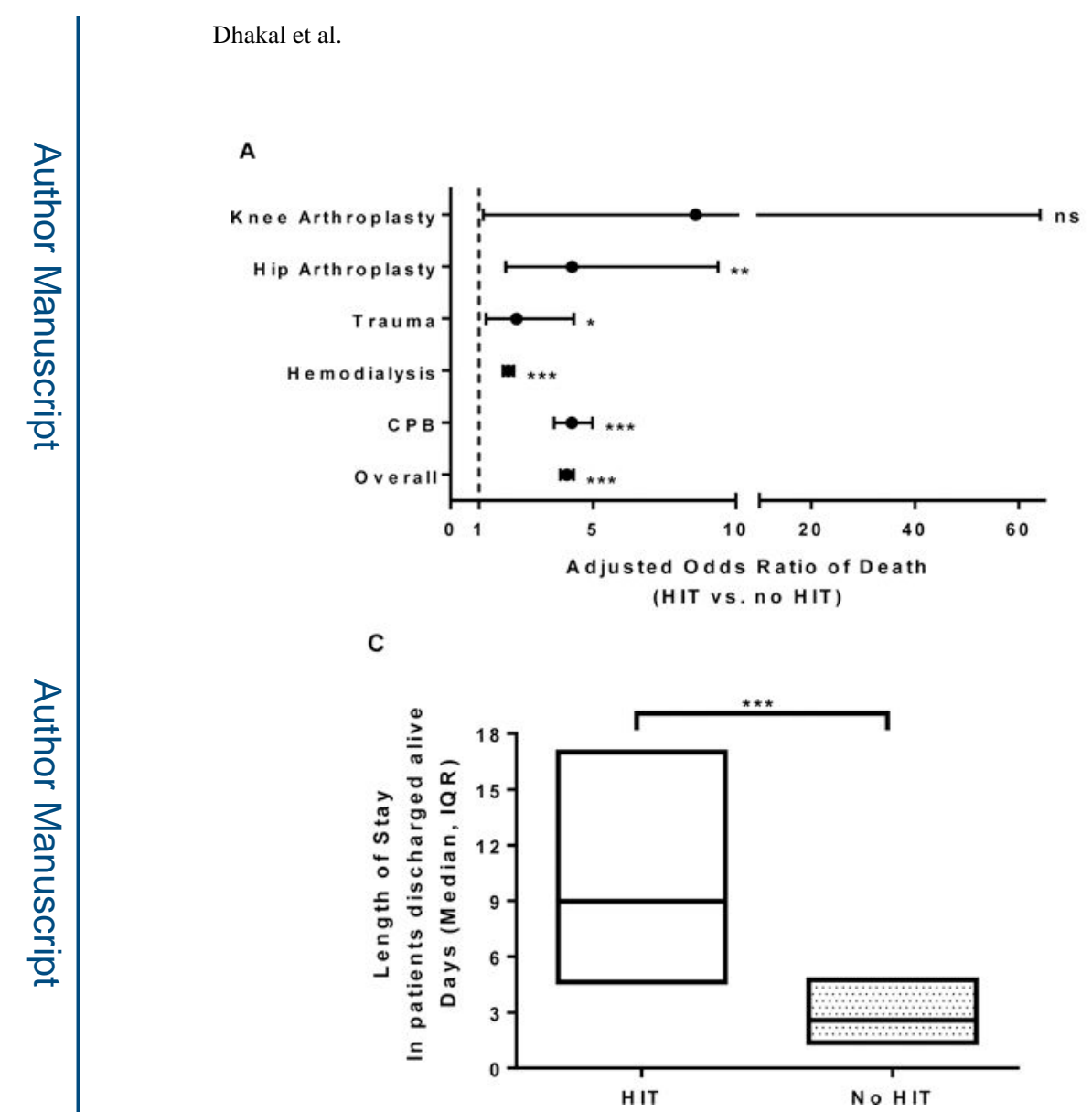

B

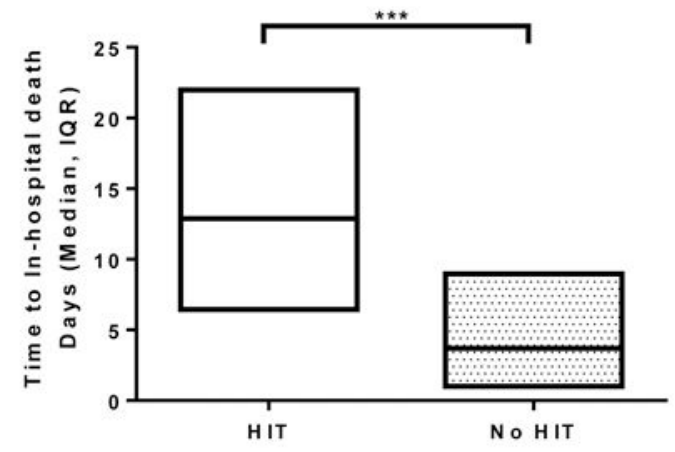

D

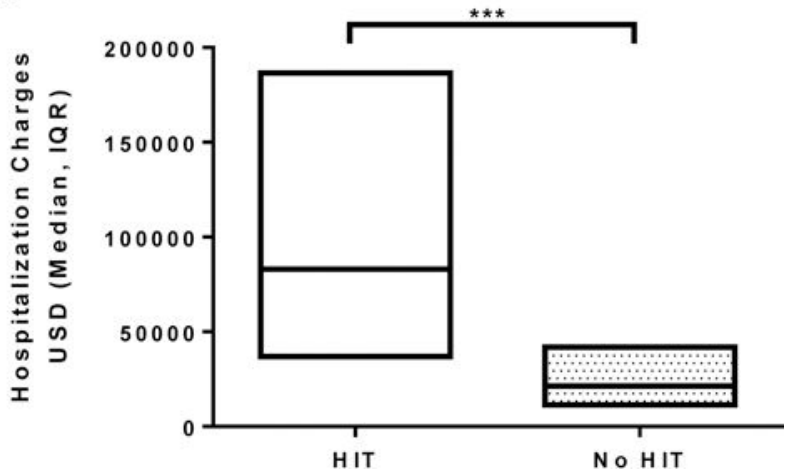

Figure 5. Incidence and time to in-hospital mortality, Length of stay and Hospitalization Charges.

(A) Adjusted odds ratio of death is high in HIT discharges. The gingival/ periodontal disease subgroups had too few events to be included in the analysis. (B) Time to in-hospital death in HIT vs. non-HIT discharges. Abscissa denotes HIT or non-HIT discharges. The ordinate depicts median time to death and interquartile range (IQR). (C) Length of stay. The ordinate depicts median length of stay and IQR. (D) Hospitalization charges. The ordinate depicts median hospitalization charges and IQR. ***,** and * denote $\mathrm{p}<0.0001$, $\mathrm{p}<0.001$ and $\mathrm{p}<0.01$, respectively. ns-not significant 
Table 1.

\section{Patient demographics.}

p values (univariate analysis) were calculated using survey-weighted Chi-squared tests for categorical variables and survey-weighted ANOVA for continuous variables. No missing data were noted for age or race.

\begin{tabular}{|c|c|c|c|}
\hline Characteristic & HIT & No HIT & p-value \\
\hline All discharges (n) & 97566 & 149911247 & \\
\hline Cardiopulmonary bypass (n) & 7702 & 1222660 & \\
\hline Hemodialysis (n) & 23012 & 4885088 & \\
\hline Trauma/Injury (n) & 541 & 602402 & \\
\hline Hip arthroplasty (n) & 845 & 1942508 & \\
\hline Knee arthroplasty (n) & 676 & 3021927 & \\
\hline Gingival/periodontal disease (n) & 106 & 88515 & \\
\hline Median age(SE), yrs & $66.4(0.2)$ & $58.2(0.1)$ & $<0.001$ \\
\hline \multicolumn{4}{|l|}{ Sex } \\
\hline Weighted (n) & 97566 & 149802092 & \\
\hline Missing Data (n) & 0 & 109154 & \\
\hline Female \% (SE) & $49.63(0.4)$ & $59.52(0.1)$ & $<0.001$ \\
\hline \multicolumn{4}{|l|}{ Race \% (SE) } \\
\hline White & $62.3(0.8)$ & $62(0.5)$ & \\
\hline Black & $15.5(0.6)$ & $13.3(0.3)$ & $<0.001$ \\
\hline Hispanic & $8.5(0.5)$ & $9.8(0.3)$ & \\
\hline Other/Unknown & $13.8(0.7)$ & $15(0.5)$ & \\
\hline \multicolumn{4}{|l|}{ Teaching Hospital Status } \\
\hline Weighted (n) & 56125 & 70055232 & \multirow{3}{*}{$<0.001$} \\
\hline Missing Data (n) & 1011 & 1290676 & \\
\hline$\%(\mathrm{SE})$ & $58.1(1.0)$ & $47.1(0.4)$ & \\
\hline
\end{tabular}

